\title{
A Conversation with John Sunderland, Johannes Czernin, and Thomas Hope
}

John J. Sunderland ${ }^{1}$, Johannes Czernin ${ }^{2}$, and Thomas Hope ${ }^{3}$

${ }^{1}$ University of Iowa, Iowa City, Iowa; ${ }^{2}$ David Geffen School of Medicine at UCLA, Los Angeles, California; and ${ }^{3}$ University of California, San Francisco, California

Tzer ohn J. Sunderland, PhD, MBA, was interviewed by Johannes Czernin, MD, editor in chief of The Journal of Nuclear Medicine, and by Thomas Hope, MD, an associate professor and director of Molecular Therapy for the Molecular Imaging and Therapeutics Clinical Section in the Department of Radiology and Biomedical Imaging at the University of California, San Francisco (UCSF). Sunderland is an associate professor of radiology, with secondary appointments in radiation oncology, as well as physics and astronomy, at the University of Iowa Carver College of Medicine/ University of Iowa Health care (Iowa City). He is also director of the PET Imaging Center. He currently serves as cochair of the SNMMI Clinical Trials Network and as chair of the Radiologic Society of North America's Quantitative Imaging Biomarker Alliance FDG/PET Biomarker Committee. Sunderland's widely recognized work spans a broad scope of PET and nuclear medicine applications. This discussion focused on his work in advancing ${ }^{68} \mathrm{Ga}$-DOTATOC for neuroendocrine tumor imaging through the Food and Drug Administration (FDA) review and approval process.

Dr. Czernin: John, Tom and I appreciate that you are taking the time to talk to our readers. Together with the Iowa team, you have recently successfully completed a new drug application (NDA) for ${ }^{68} \mathrm{Ga}$-DOTATOC. Tell us a little about the history of the DOTATOC project.

Dr. Sunderland: We have a strong neuroendocrine tumor program ongoing here, which has been driven by our medical oncologist/ endocrinologist team for the last 30 years: Sue and Tom O'Dorisio. People from all over the country have come here. So we started the DOTATOC project some time ago and actually did small ${ }^{90} \mathrm{Y}$ therapy studies quite early on.

Dr. Czernin: These were done under an investigational new drug (IND) application?

Dr. Sunderland: Yes. Then Mike Schultz came on board about a decade ago and started work with ${ }^{68} \mathrm{Ga}$ generators. We did reproducibility studies under the FDA's Radioactive Drug Research Committee and wrote our first IND and looked at safety and efficacy, because we had our eyes on potentially getting an NDA. In our second and third IND protocols, we compared ${ }^{68} \mathrm{Ga}$-DOTATOC with ${ }^{111}$ In-octreotide and also looked at changes in management prompted by the PET scan. That was at about the time that Advanced Accelerator Applications started to develop DOTATATE. We put our development plans on hold, because DOTATOC and DOTATATE were functionally very similar. Thus, we thought that if they got an NDA for DOTATATE there might have been no room

COPYRIGHT @ 2020 by the Society of Nuclear Medicine and Molecular Imaging. for DOTATOC. But then the FDA Office of Orphan Product Development ruled that DOTATATE and DOTATOC were distinct molecules. So we went from there.

Dr. Hope: When DOTATATE was approved, what made you think that you should still go with DOTATOC? What was the value of DOTATOC when DOTATATE had been approved?

Dr. Sunderland: At that point, we were well aware of the cost of radio-

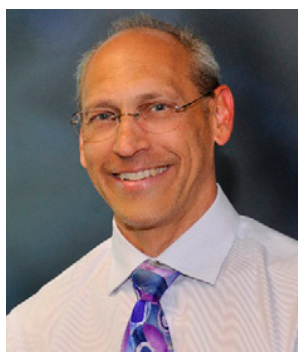

John J. Sunderland, PhD, MBA pharmaceuticals. When these compounds go off Centers for Medicare and Medicaid Services pass-through reimbursement after 2 or 3 years, reimbursement drops dramatically. The costs of these drugs then must be carried by hospitals or outpatient clinics at a substantial loss. We looked into our crystal ball and decided that, because we wanted to continue to provide services to the neuroendocrine patient community, we needed to find a way to do it at a lower production cost.

Dr. Czernin: When did you initiate contact with the FDA for your NDA? How long did the process take?

Dr. Sunderland: Our initial meeting with FDA was a pre-IND meeting in 2015, at which we discussed strategy for using both literature and our IND data from our 3 trials to support an NDA. Then, during our pre-NDA meeting in 2017, the FDA agreed that our clinical trial data were likely sufficient to apply for an NDA. But they also suggested that we conduct and publish a formal metaanalysis as an additional supporting trial.

Dr. Hope: Your pre-IND meeting was in 2015 and your preNDA meeting happened in 2017. What happened in those 2 years?

Dr. Sunderland: This was a real education to me. We learned that harmonizing our clinical trials was critical. Rather than using our 3 prospective clinical trials as they had been designed, we changed the rules. We designed an entirely new retrospective study on our prospectively collected data. We established a single reference standard for all 3 trials and collected the data for all in the same way. We reread all the images with 2 physicians on the same case report forms and then combined the retrospective analyses of the 3 prospective trials to form the basis for the NDA.

Dr. Hope: Along the same lines, we report a lot of data in the literature and much of it is not usable in the setting of approvals. How should we publish our work to better support potential registration of drugs later on?

Dr. Sunderland: We had the grim realization that the quality of the nuclear medicine literature is not as high as it should be. Too 
many of our studies are retrospective. Prospective trials on the front end are needed, and this requires funding. But, second, the reporting of the data in the trials is not as good or as consistent as it could be. Adverse event reporting is critical for demonstration of safety. Reporting of reference standards is often missing or not rigorous enough.

Dr. Czernin: The problem is that, as you said, the studies are unfunded. Therefore, almost all of them will be retrospective. When you have industry involvement, with their knowledge of all the regulatory issues, the design, analysis, and reporting will be better. The only exception is the IND with cost recovery, where you can charge patients for the cost of the study drug.

Dr. Sunderland: I agree wholeheartedly. However, the cost recovery mechanism is only available late in the development process. I do believe that the National Cancer Institute (NCI) is missing the boat here. The NCI is stressing translation into real clinical practice. It would seem to me that with a few well-chosen million dollars for academically sponsored late-phase clinical trials, they could help some of the nonproprietary PET radiopharmaceuticals to the finish line.

Dr. Hope: I'm assuming your 3 trials were funded via cost recovery. The issue here is that only patients who can afford it will be able to participate in the trials. Did your Institutional Review Board (IRB) push back, and how much trouble was implementing cost recovery?
Dr. Sunderland: I would argue that there is a very healthy pipeline of commercial PET radiopharmaceuticals out there. There seems to be a financial model in which businesses can do well. I wish the reimbursement would get more rational, predictable, and sustainable for longer periods. However, some nonproprietary PET radiopharmaceuticals are still out there, and I suspect there will be some in the future. The question is how does one help move those to regulatory approval? The NDA process itself is daunting, complex, and expensive. But if we all share data and work unselfishly, the next one will be much easier. Doing large-scale prospective trials across sites via a clinical trials network would be very helpful. A harmonized approach to data collection is mission critical, however. The Australians have put together such a network with seamless coordination and infrastructure that allows for efficient data collection across sites. It seems to be a model we should emulate.

Dr. Czernin: John, in addition to cost recovery money, did you ever calculate how much your department invested to get the NDA done?

Dr. Sunderland: We spent about $\$ 400,000$ out of pocket. We were supported by the Margie and Robert E. Petersen Foundation and are very thankful for that. Eighty-five percent of the money was spent on consultants.

Dr. Hope: This does not include your time, the physicians' time for reading the scans, the clinical research coordinators, everything needed to actually perform all the studies and get everything prepared for FDA submission.

\section{"I've been incredibly impressed with the fine line that the FDA is walking. They remain uncompromising in their standards and their approach, but they were incredibly supportive throughout the process. It kind of felt that they were rooting for us while at the same time doing their job within the rules."}

Dr. Sunderland: Funding came in part from the National Institutes of Health, but a major portion came from the IND with cost recovery. Interestingly, the IND with cost recovery for DOTATOC went through IRB approval relatively smoothly. We set up a special financial counseling pathway for patients and tried to make payment as affordable and flexible as possible. It was only when we tried to implement the IND with cost recovery for prostatespecific membrane antigen (PSMA) assessment that we started getting pushback from our IRB. You know, the various review bodies have different perspectives-our IRB was concerned that only people who could afford to have this scan could participate. Ultimately, however, it was approved.

Dr. Czernin: Do you share our impression that the FDA is actually extremely supportive of what you were trying to do with DOTATOC, like what Tom initiated and did together with us for PSMA imaging?

Dr. Sunderland: Absolutely. I've been incredibly impressed with the fine line that the FDA is walking. They remain uncompromising in their standards and their approach, but they were incredibly supportive throughout the process. It kind of felt that they were rooting for us while at the same time doing their job within the rules. They are remarkable, well-meaning professionals who are trying to do their job.

Dr. Hope: Do you think that NDAs can become something that the academic community can do in general? And if so, how should we in the academic world think about applying your experience to new agents?
Dr. Sunderland: That's right, and it was a huge effort. I shudder to calculate the person hours that were associated with it over the 3 years of preparation.

Dr. Hope: How does your NDA help smaller hospitals or other academic centers to start up their ${ }^{68}$ Ga-DOTATOC imaging services? What can they do, and what do they need to do?

Dr. Sunderland: First, we obviously waived exclusivity as part of our application, so anybody can now apply for an ANDA. We will provide anyone who asks with the relevant portions of the chemistry, manufacturing, and controls section. The challenge with submitting an ANDA is that it still may be on the order of 2 years before ANDA approval, because applications go through the back-logged FDA Office of Generic Drugs. We are supporting a company that is interested in marketing a DOTATOC kit. If they were to submit an NDA for their kit then, on approval, anybody could use DOTATOC sooner, with the kit.

Dr. Hope: Can you briefly address the gallium generator shortage. Is that going to be a problem for centers who might want to do this? And if a kit were available, how would that actually work in terms of implementation?

Dr. Sunderland: I may be wrong, but I think that the Good Manufacturing Practice generator shortage is maybe a thing of the past. Manufactured supply is much higher now. The alternative option is that we're working on submitting an amendment to our NDA for cyclotron-produced gallium so that sites with a cyclotron could invest in a target and produce ${ }^{68} \mathrm{Ga}$ on site. 
Dr. Hope: The neuroendocrine tumor patient community is very strong. How important was this group in helping to push your process forward?

Dr. Sunderland: The patient community has been indirectly supportive. Josh Mailman, as a spokesman and representative of the community, has been amazing. What was driving our effort was that we wanted to provide access for our patients in the Midwest and around Iowa. Patient-centered care and passion were at the heart of our NDA effort. Tom, was the prostate cancer community supportive of your efforts?

Dr. Hope: In the prostate world the patients are interested but not engaged to the level of the neuroendocrine community. There were other drivers: In 2013, during a theranostics symposium at Johns Hopkins, Lou Marzella from the FDA in essence said, "Send me an NDA." That had a huge impact on my understanding and belief that the FDA would be very receptive to these types of things.

Dr. Czernin: The Prostate Cancer Foundation does play a very important role. They did not directly fund the ${ }^{68} \mathrm{Ga}-\mathrm{PSMA} N \mathrm{NA}$, but they supported both the UCSF and University of California Los Angeles programs with major awards for different research.

Dr. Hope: Let's get back to business for a moment: John, did you have an option to sell your data to commercial entities, and, if so, why didn't you?
Dr. Sunderland: We were asked to do that, and we opted not to. This was a University of Iowa decision. So, it wasn't my decision, although I agreed with it. But that said, it's a perfectly reasonable thing to do for an academic site that has valuable clinical data, if commercial collaboration will help get the product to the market.

Dr. Hope: As you are no longer writing an NDA, what is next for you?

Dr. Sunderland: I would like to throw my energy and efforts toward personalized dosimetry in theranostics. This is so obviously the right thing to do. I think dosimetry might play a central role in getting theranostics right. Nuclear medicine is a really exciting place to be, particularly for someone who's been in it for a while. My first 15 years in PET were mired in clinical acceptance and reimbursement and regulatory chaos. What followed for the next 10 years was clinical FDG on cruise control. But now things are really exciting. New disease-specific PET agents are in the pipeline. Targeted radiotherapy (and associated imaging) is exploding. So many options for future work. It's hard to decide what to do next. You have to be selective.

Dr. Czernin: John, Tom and I are very grateful for your time, and JNM readers will appreciate the work you and the Iowa team have done to make ${ }^{68} \mathrm{Ga}$-DOTATAOC available to patients with neuroendocrine tumors. 